\title{
Graft Versus Tumour Effect
}

\author{
Mairéad NíChonghaile
}

\begin{abstract}
The treatment of relapsed disease remains challenging, and it is well accepted that concept of allogeneic HSCT relies upon both the conditioning or preparative regimen used for the recipient and the graft versus malignancy $(\mathrm{GvM})$ or leukaemia $(\mathrm{GvL})$ effect provided by the donor $\mathrm{T}$ cells and NK cells. Strategies which involve harnessing this effect are crucial to success and need to be exploited and refined to improve outcome. Further research is required to identify new strategies and therapies to improve the outlook for patients who relapse post-HSCT.
\end{abstract}

The nursing challenges following relapse are immense; the psychological support required is complex and largely falls to the nurse to coordinate and deliver regardless of the selected treatment approach.

\section{Keywords}

Graft versus malignancy $(\mathrm{GvM})$ or leukaemia $(\mathrm{GvL}) \cdot$ Donor lymphocyte infusions (DLI) • Relapse • Chimerism

\subsection{Introduction}

It is well accepted that concept of allogeneic haematopoetic stem cell transplant (HSCT) relies upon both the conditioning or preparative regimen used for the recipient and the graft versus malignancy $(\mathrm{GvM})$ or leukaemia $(\mathrm{GvL})$ effect provided by the donor $\mathrm{T}$ cells and NK cells. The autoim-

\footnotetext{
M. NíChonghaile

HOPE Directorate, St James's Hospital, Dublin, Ireland

e-mail: maireadnichonghaile@eircom.net
}

mune attack on the malignancy helps to eradicate the disease in the recipient with the aid of the condition regimen. The susceptibility of a malignant condition being eradicated by GVM of GvL effect varies with the most sensitive conditions being chronic myeloid leukaemia, chronic lymphocytic leukaemia, low-grade B-lymphoproliferative disorders, mantle cell lymphoma and EBV lymphoproliferative conditions. Most other conditions have an intermediate sensitivity to the GvM or GvL effect with conditions that have a special proliferation or that are advanced or chemo-refractory having the least response. 


\subsection{Mechanism of GvM/GvL Effect}

Both T lymphocytes and NK cells participate in the GvL effect, and it is believed that cytotoxic $\mathrm{T}$ cells recognise several classes of antigens on leukaemic cells. The NK cells target MMag selfproteins (present on the tissues of the recipient) which are overexpressed by the leukaemia, e.g. proteinase 3 and elastase tumour-specific antigens, e.g. Wilms tumour 1 , and fused proteins, e.g. BCR-ABL, by using the perforin-granzyme pathway to kill their targets. However they are only activated when inhibitory signals from self (recipient) MHC class 1 molecules on the target are missing or overcome by activating signals through the NKG2D receptor. The suppressor of the recipient's immune system allows the donor cells to provoke this effect.

\subsection{Minimal Residual Disease (MRD)}

The purpose of monitoring MRD in the posttransplant setting is to track disease response or remission or low-level disease recurrence when the quantity of a particular marker starts to increase. This enables a therapeutic intervention to be implemented very early and may optimise the chance of success.

MRD can be monitored using molecular methods - this is where the underlying condition has a specific market or protein that can be monitored by using flow cytometry. Table 12.1 shows the cytogenetic abnormalities that can be targeted in some diseases (page 410 Treleaven and Barrett 2009), and Table 12.2 shows some of the molecular targets (Apperly et al. 2012) if they were present at diagnosis.

\subsection{Chimerism}

Chimerism analysis is another important tool in the post-HSCT follow-up of the recipient. It demonstrates the degree of engraftment of donor
Table 12.1 Common targets for MRD screening in different malignancies

\begin{tabular}{|l|l|}
\hline $\begin{array}{l}\text { Disease } \\
\text { Myelodysplastic syndromes }\end{array}$ & $\begin{array}{l}\text { MRD target } \\
\text { trisomy } 8\end{array}$ \\
\hline Chronic myeloid leukaemia & $\mathrm{t}(9 ; 22)$ \\
\hline $\begin{array}{l}\text { Acute myeloid leukaemia } \\
\text { Acute lymphoblastic }\end{array}$ & $\mathrm{t}(8 ; 21)$; inv $(16)$ \\
\hline leukaemia & $\mathrm{t}(4 ; 11) ; \mathrm{t}(8 ; 14)$ \\
\hline Follicular lymphoma & $\mathrm{t}(14 ; 18)$ \\
\hline Mantle cell lymphoma & $\mathrm{t}(11 ; 14)$ \\
\hline $\begin{array}{l}\text { Chronic lymphocytic } \\
\text { leukaemia }\end{array}$ & $\begin{array}{l}\operatorname{del}(13 \mathrm{q}), \operatorname{del}(11 \mathrm{q}) ; \\
\operatorname{del}(17 \mathrm{p})\end{array}$ \\
\hline Multiple myeloma & $\operatorname{del}(13 \mathrm{q}), \operatorname{del}(11 \mathrm{q})$ \\
\hline
\end{tabular}

Table 12.2 Examples of Molecular targets in different malignacies

\begin{tabular}{|l|l|} 
Disease & Molecular target \\
\hline B-ALL & $\begin{array}{l}\text { TEL-AML1 } \\
\text { BCR-ABL1 } \\
\text { Ig/TCR gene rearrangements }\end{array}$ \\
\hline T-ALL & $\begin{array}{l}\text { Ig/TCR gene rearrangements } \\
\text { Tald1 }\end{array}$ \\
\hline APML & PML-RARA \\
\hline AML & $\begin{array}{l}\text { AML1-ETO } \\
\text { CBFb-MYH11 } \\
\text { WT-1 } \\
\text { NPM1 mutated } \\
\text { FIT3 }\end{array}$ \\
\hline
\end{tabular}

cells and offers the possibility to identify impending graft rejection and can also be an indicator of disease relapse or recurrence. Chimerism can also be used as a basis for treatment intervention to prevent graft rejection and maintain engraftment and is used as a mechanism to administer pre-emptive immunotherapy to provoke the GvM or GvL effect particularly in high-risk patients.

Chimerism allows the monitoring of the ratio of donor- and recipient-derived cells in nongenetically identical donor and recipient pairs. This allows for timely intervention in the care of the recipient. The term "chimerism" comes from Greek mythology where the chimera was a firespitting monster with the head of a lion, body of a goat and the tail of a serpent and is used to describe the fact of two entities - DNA from two people - existing in the one person. 
Initially it was felt that in order for all HSCT to be considered successful, an individual would have to be $100 \%$ donor chimerism, and this is certainly the case with malignant conditions. However, in non-malignant conditions (e.g. aplastic anaemia or haemoglobinopathies), a mixed chimerism may be enough to restore normal haematopoiesis.

While the total (unfractionated) chimerism result is important, more information can be elicited if lineage-specific chimerism is performed allowing separate tracking of lymphoid and myeloid engraftment and in itself can provide very useful information about possible disease relapse. This should be used in conjunction with other means of MRD analysis and diagnosis.

Schedule and protocols for chimerism analysis are centre, disease and treatment specific, and reference should be made to your institution's policy.

\subsection{Management of Relapsed Disease}

When a recipient has evidence of MRD or a decrease in chimerism, there are a number of strategies that can be followed. In the case of mixed chimerism, the schema in Fig. 12.1 can be followed.

Relapse usually occurs in the BM but may also be at extramedullary sites, thought to be due to immune escape from patrolling lymphocytes. It is also thought that leukaemia can escape from the immune control provided by the donor's T cells by mutating or becoming a more resistant clone of the original disease. The cause of the relapse may also be different in different haematological malignancies. While relapses can occur many years after HSCT suggesting that perhaps the underlying disease was never eradicated and was held in control by the donor immune system, it is more common with CML, and in this disease, the administration of DLI can often restore remission.

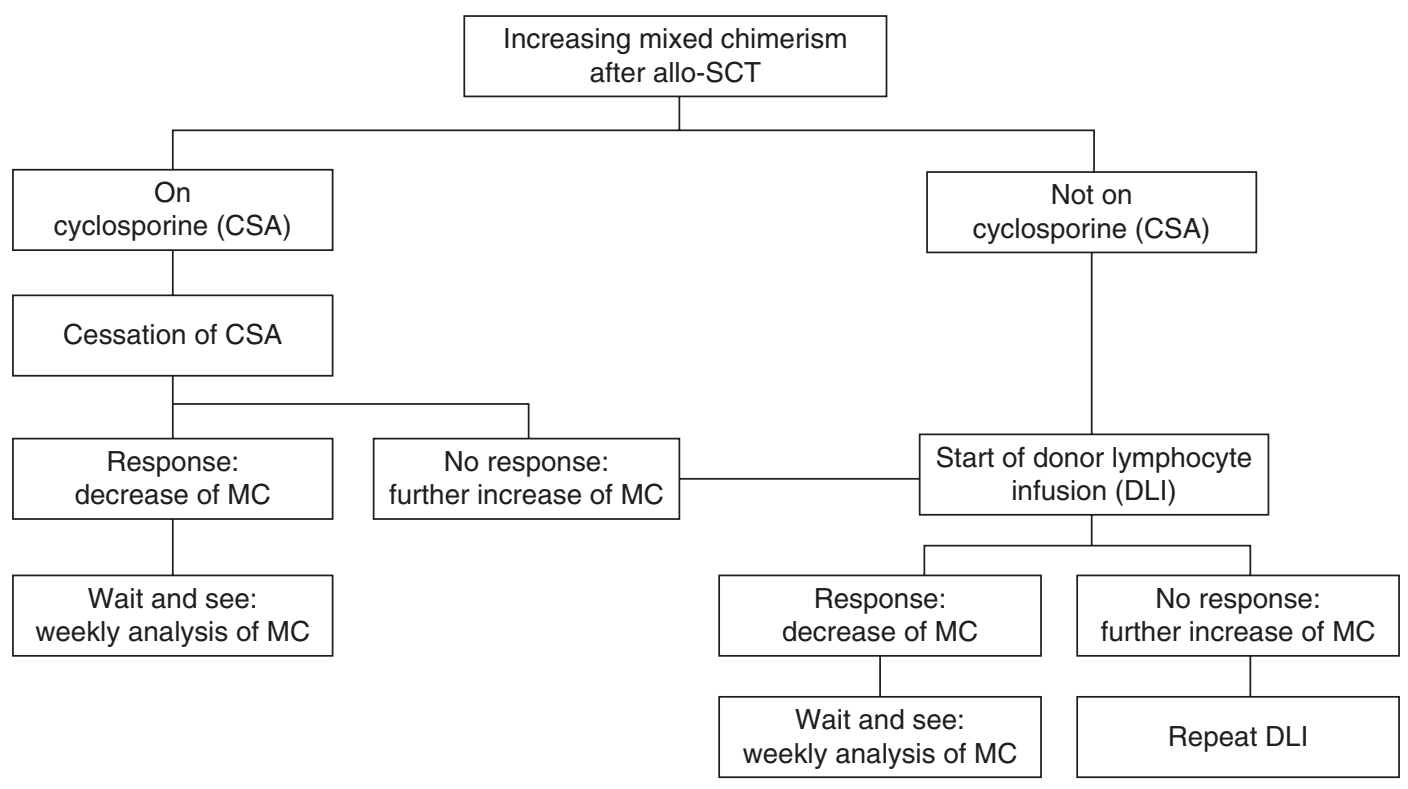

Fig. 12.1 Patients with increasing MC post transplant (5\% or more autologous cells) compared to the previous sample are offered further therapy. Immunotherapy for patients receiving CSA consists of immediate discontinuation of the immunosuppressive agent. Chimerism is then assayed weekly until CC status is restored. If MC continues to increase after cessation of CSA, a DLI is given. Immunotherapy for patients not receiving CSA consisted of DLI as frontline treatment. The cell dose administered is based on the number and potential severity of HLA mismatches between the donor and recipient, and starting doses range from $2.5 \times 10^{4}$ to $1 \times 10^{6} / \mathrm{kg} \mathrm{BW}$. After DLI, chimerism status is assayed weekly until CC status is restored. Patients who show a further increase in $\mathrm{MC}$ are given an additional DLI after at least 3 weeks have elapsed. If no GVHD occurs, the dose of DLI is doubled (Bader et al. 2005) 
However the outlook for the recipient who relapses post-HSCT is poor and requires frank discussion with the recipient and their family to define the likely outcomes and success. Care for the relapsed patient revolves around five potential strategies:

- Immunotherapy

- Chemotherapy

- Molecular targeting

- Supportive care

- Palliative care

The timing of relapse is extremely important. Early relapses are difficult to treat, and any intervention may be challenging for the patient or precluded due to the proximity to the HSCT and complications experienced by the recipients. Patients who relapse after HSCT may find it extremely difficult to adapt to and accept the fact that further treatment may not be possible or may be ineffective particularly if they have already received intensive treatment prior to HSCT.

However, it is important to provide ongoing support so that patients do not feel abandoned at this stage, and this helps patients to maintain realistic levels of hope and optimism. Palliative and supportive care measures are valid and realistic options to help maintain a good quality of life and should not be ignored. The help and support of the medical team, recipient's local doctor, referring hospital and often primary care services, e.g. GP and hospice, are essential in the management and treatment of the relapsed patient. Referral to psychology, counselling or psychiatric teams may also benefit the patient and their family.

\subsection{Treatment Options for Post- allogeneic HSCT Relapse}

\author{
Immunotherapy
}

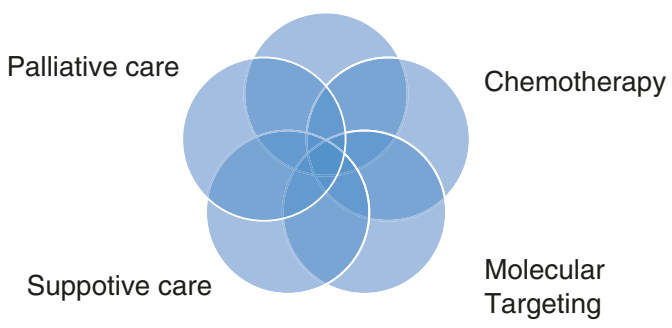

\subsection{Management of Relapsed Disease}

Immunotherapy This is an important tool in the management of the relapsed patient and can range from withdrawal of immunosuppression (if the patient is still on it) to the administration of donor lymphocyte infusions (DLI) infusions in incremental doses.

\subsection{Withdrawal of Immunosuppression}

The GvL or GvM effect of HSCT can be enhanced by reducing and stopping the IS (immunosuppressive) agent that the patient may be taking. Nursing care and education is essential in this circumstance as along with GvL the patient needs to be monitored for the development of GVHD which is extensively discussed in another chapter. 


\subsection{Responsiveness to DLI} (Adapted from Treleaven and Barrett 2009)

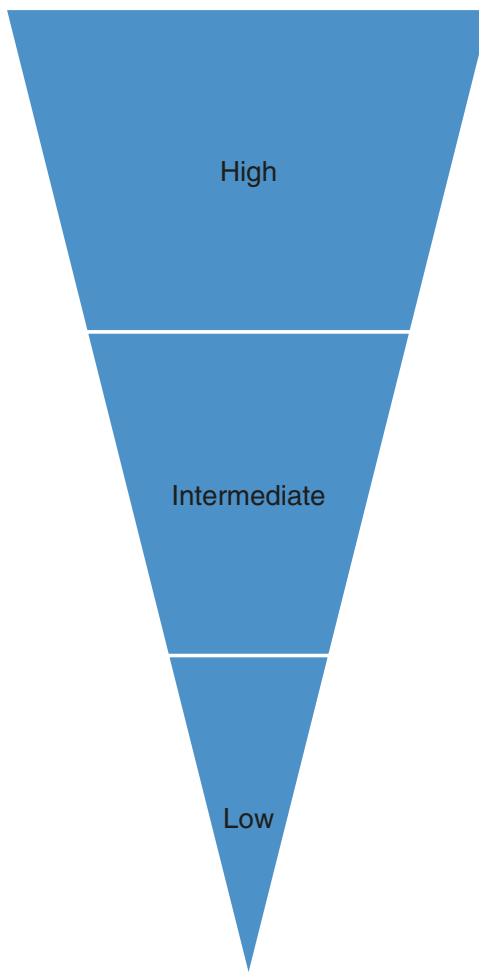

\subsubsection{DLI}

DLI alone can induce permanent remission of the underlying disease particularly in the case of CML where relapses occur at a molecular level. In other diseases, the response can be varied from effective to ineffective (Fig. 4). Most centres adopt an approach with the administration of graduated doses between 1 $\times 10^{6} \mathrm{CD} 3$ cells per $\mathrm{kg}$ and $1 \times 10^{8}$ per $\mathrm{kg}$ recipient body weight depending on time from transplant, patient performance status and type of donor.

DLI may also be administered as an adjuvant therapy to chemotherapy or targeted therapies to augment the effect of that treatment or sustain a remission if achieved.
Chronic Myeloid Leukaemia

Chronic Lymphocytic Leukaemia

Mantle Cell Lymphoma

Follicular Lymphoma

Hodgkin's Disease

Multiple Myeloma

Myelodysplastic Syndrome

Acute Myeloid Leukaemia

Acute Lymphoid Leukaemia

High Grade Non-Hodgkins Lympohma

\subsubsection{Chemotherapy}

This may be used to palliate the patient, to attempt to reduce the disease burden to facilitate DLI or targeted therapy or to achieve a remission. Patients relapsing within 6 months of HSCT often require reduced or modified dosing due to the toxicity of previous treatment or having reached the dosing limits of particular chemotherapeutic agents. Regimens for relapsed patients tend to be patient specific, and there are currently very few standardised approaches. The nursing care of these patients has been well documented in previous chapters. 


\subsection{Molecular or Targeted Therapies}

Consideration needs to be given to the administration of molecular or targeted therapies to patients who relapse post-HSCT if they are available. Disease-specific monoclonal antibodies, e.g. brentuximab, in some lymphomas; anti-CD33 agents, e.g. gemtuzumab, in myeloid malignancies; and TKIs in BCR-ABL-positive malignancies can have an important role in this setting.

\subsection{Second HSCT}

If a patient experiences a relapse later postHSCT, consideration may be given to a second HSCT using stem cells either from the original donor or an alternative donor. In malignant disease, usually a second procedure is only feasible when remission has been achieved following successful administration of chemotherapy, a molecular or targeted therapy. The morbidity and mortality associated with a second HSCT is often significant, and the patient and family should be carefully counselled before such an undertaking.

\section{Conclusion}

The treatment of relapsed disease remains challenging, and further research is required to identify new strategies and therapies to improve the outcome for this group of patients.

The nursing challenges are immense, and the psychological support required is complex and largely falls to the nurse to coordinate and deliver regardless of the subseque nt treatment approach. The nursing team will continue to support patients and their families and help them to adjust to the changes ahead. The demands on the team can mean that it is difficult to take the time to reflect and deal with the changing care and focus for the patients. The adjustments and difficulties experienced in caring for the relapse patient need to be matched by a team that continues to support each other.

\section{References}

Apperly J, Carreras E, Gluckman E, Masszi T. Haematopoietic stem cell transplantation. 6th revised ed. Genoa: Forum Service; 2012

Bader P, Niethammer D, Willasch A, Kreyenberg H, Klingebiel T. How and when should we monitor chimerism after allogeneic stem cell transplantation. Bone Marrow Transpl. 2005;35:107-19.

Treleaven J \& Barrett AJ 2009 Haematopoietic stem cell transplantation in clinical practice. Churchill Livingstone Elsevier; Edinburgh

Open Access This chapter is licensed under the terms of the Creative Commons Attribution 4.0 International License (http://creativecommons.org/licenses/by/4.0/), which permits use, sharing, adaptation, distribution and reproduction in any medium or format, as long as you give appropriate credit to the original author(s) and the source, provide a link to the Creative Commons license and indicate if changes were made.

The images or other third party material in this chapter are included in the chapter's Creative Commons license, unless indicated otherwise in a credit line to the material. If material is not included in the chapter's Creative Commons license and your intended use is not permitted by statutory regulation or exceeds the permitted use, you will need to obtain permission directly from the copyright holder. 